\title{
Prevalência de sobrepeso e obesidade e fatores associados em mulheres de São Leopoldo, Rio Grande do Sul: um estudo de base populacional
}

\author{
Prevalence of overweight and obesity and associated \\ factors in women of São Leopoldo/RS - Population-based study
}

\author{
Josiele Flores Lisowski ${ }^{1}$ (D), Heloísa Marquardt Leite ${ }^{1}$ (D), Fernanda Bairros² (D), \\ Ruth Liane Henn ${ }^{1}$ (D) Juvenal Soares Dias da Costa ${ }^{1}$ (D), \\ Maria Teresa Anselmo Olinto ${ }^{1}$
}

\begin{abstract}
Resumo
Introdução: O sobrepeso e a obesidade são multicausais, porém seus determinantes ainda não foram completamente estudados. Objetivo: Estimar a prevalência de sobrepeso e de obesidade e os fatores associados. Método: Estudo transversal de base populacional com 981 mulheres (20 a 60 anos) de São Leopoldo/RS. O peso e a altura foram aferidos para o cálculo do Índice de Massa Corporal (IMC). Mulheres com IMC entre 25,0 e 29,9 kg/m² e IMC $\geq 30,0 \mathrm{~kg} / \mathrm{m}^{2}$ foram classificadas com sobrepeso e obesidade, respectivamente. As razões de prevalência e os intervalos de confiança de $95 \%$ foram estimados por meio de regressão de Poisson. Resultados: A prevalência de sobrepeso foi de 33\% (IC95\%: 30,1-36,0) e de obesidade foi de 31,2\% (IC95\%: 28,3-34,1). Após ajuste, maiores probabilidades de sobrepeso ocorreram em mulheres mais velhas, pertencentes às classes econômicas mais baixas, casadas ou em união, com histórico de obesidade materna e com uma ou mais gestações. As maiores probabilidades de obesidade ocorreram em mulheres mais velhas, de baixa renda, com histórico de obesidade materna e paterna e com três ou quatro gestações. Menarca acima de 12 anos e prática de atividade física de lazer foram fatores de proteção para a obesidade. Conclusão: Esses resultados evidenciam a necessidade de ações de prevenção de longo prazo, algumas delas envolvendo ações transgeracionais.
\end{abstract}

Palavras-chave: sobrepeso; obesidade; mulheres; prevalência; estudos transversais.

\begin{abstract}
Background: Overweight and obesity are multicausal; however, their determinants have not been fully studied. Objective: To estimate the prevalence of overweight and obesity and associated factors. Method: A population-based cross-sectional study with 981 women (20-60 years) from São Leopoldo/RS was carried out. Weight and height were measured by the calculation of Body Mass Index (BMI). Women with a BMI between $25.0-29.9 \mathrm{~kg} / \mathrm{m}^{2}$ and a BMI $\geq 30.0 \mathrm{~kg} / \mathrm{m}^{2}$ were classified as overweight and obese, respectively. Prevalence ratios and $95 \%$ confidence intervals were estimated using Poisson regression. Results: The prevalence of overweight was $33.0 \%(95 \% \mathrm{Cl}: 30.1-36.0)$ and obesity was $31.2 \%(95 \% \mathrm{Cl}$ : 28.3-34.1). After adjustment, higher probabilities of overweight occurred in older women, from lower economic classes, married or in union, with a history of maternal obesity and

${ }^{1}$ Programa de Pós-graduação em Saúde Coletiva, Universidade do Vale do Rio dos Sinos (Unisinos) - São Leopoldo (RS), Brasil.

${ }^{2}$ Departamento de Assistência e Orientação Profissional, Escola de Enfermagem, Universidade Federal do Rio Grande do Sul (UFRGS) - Porto Alegre (RS), Brasil. Trabalho realizado na Universidade do Vale do Rio dos Sinos (Unisinos) - São Leopoldo (RS), Brasil.

Endereço para correspondência: Maria Teresa Anselmo Olinto - Programa de Pós-graduação em Saúde Coletiva, Universidade do Vale do Rio dos Sinos (Unisinos), Av. Unisinos, 950, CP 275 - CEP: 93022-000 - São Leopoldo (RS), Brasil - Email: mtolinto@gmail.com

Fonte de financiamento: 0 estudo foi contemplado com auxílio do Conselho Nacional de Desenvolvimento Científico e Tecnológico (CNPq) pelo Edital MCTI/CNPQ/Universal 14/2014.

Conflito de interesses: nada a declarar.
\end{abstract}


with one or more pregnancies. The highest probabilities of obesity occurred in older women, from low-income, with a history of maternal and paternal obesity and with three or four pregnancies. Menarche over 12 years old and leisure-time physical activity were protective factors for obesity. Conclusion: These results highlighted the need for long-term prevention actions, some involving transgenerational actions.

Keywords: overweight; obesity; women; prevalence; cross-sectional studies.

\section{INTRODUÇÃO}

A obesidade é uma doença que se caracteriza por excesso de adiposidade corpórea, generalizada e/ou localizada, e atualmente integra o grupo das doenças crônicas não transmissíveis (DCNT) $)^{1,2}$. O sobrepeso, por sua vez, é um estágio prévio de adiposidade, que se caracteriza pelo risco de desenvolver obesidade ${ }^{3}$.

Ambos são considerados pandemias mundiais e graves problemas de saúde pública e atingem países de alta, média e baixa renda ${ }^{3,4}$. Segundo a Organização Mundial da Saúde (OMS), em todos os continentes, as mulheres são mais obesas do que os homens ${ }^{5}$.

São atribuíveis ao ganho de peso corporal $44 \%$ do diabetes mellitus, 23\% das doenças cardíacas isquêmicas e 7 a $41 \%$ dos cânceres $^{6}$. Nas mulheres também pode ocorrer infertilidade, síndrome de ovários policísticos, aumento de complicações maternas e fetais, como pré-eclâmpsia, diabetes gestacional, aborto espontâneo e, especificamente no bebê, macrossomia e defeitos do tubo neural ${ }^{7-11}$.

A prevalência de obesidade na população feminina vem apresentando um rápido e progressivo aumento nas últimas décadas em todo o mundo ${ }^{4}$. No Brasil, a prevalência de obesidade nesse grupo populacional passou de 13,1\% (2002-2003) para 16,9\% (2008-2009), conforme dados das Pesquisas de Orçamentos Familiares $(\mathrm{POF})^{12,13}$. Além disso, a prevalência nas mulheres da região Sul foi maior em relação às demais regiões do Brasil ${ }^{12,13}$.

Por ser uma doença complexa e multicausal, seus determinantes ainda não foram completamente identificados e estudados e, provavelmente, não serão compreendidos por completo, uma vez que podem ter um caráter dinâmico no tempo ${ }^{3}$. De modo geral, evidenciam-se na literatura diferenciais na determinação da ocorrência de sobrepeso e de obesidade. Em um estudo realizado em 2003 com mulheres adultas de São Leopoldo/RS, foram identificadas diferenças nas características socioeconômicas associadas a esses dois eventos: a escolaridade das participantes esteve associada apenas com o sobrepeso, e a classe econômica, apenas com a obesidade. Mas, por outro lado, foram verificadas similaridades, tais como forte associação de ambos os eventos com características da vida reprodutiva e hereditárias (número de gestações) ${ }^{14}$.

Assim, em virtude do crescente aumento do sobrepeso e da obesidade, do impacto que esses eventos podem ocasionar na saúde das mulheres e da possibilidade de acompanhamento temporal da evolução desses dois desfechos na população de mulheres de São Leopoldo/RS, insere-se a importância desta pesquisa. Desse modo, o objetivo deste artigo foi estimar a prevalência de sobrepeso e obesidade e os fatores associados em mulheres de São Leopoldo/RS.

\section{MÉTODO}

Foi realizado estudo transversal de base populacional com amostra representativa de mulheres entre 20 e 60 anos residentes na região urbana de São Leopoldo, Rio Grande do Sul. O tamanho da amostra foi calculado para um estudo maior, do qual este trabalho teve origem, para investigação de diferentes desfechos em saúde. Optou-se pelo resultado com maior tamanho de amostra, neste caso utilizando a variável exame citopatológico atrasado como desfecho. Assim, a amostra permitiu identificar uma razão de risco de 2,0 para um nível de confiança de $95 \%$ e poder estatístico de $80 \%$, mantida a razão de não expostos por expostos de 1:2 para a variável escolaridade. Considerando possíveis perdas/recusas e controle de fatores de confusão na análise dos dados, a amostra foi acrescida em $25 \%$, totalizando 1.281 mulheres.

Foi realizada amostragem por múltiplos estágios, em que, primeiramente, foram sorteados, de forma sistemática, 45 setores censitários entre os 371 da região urbana da cidade. Posteriormente, em cada setor selecionado, foram sorteadas as quadras e a esquina, e a partir desta foi identificado o domicílio para início da coleta de dados. As casas foram alternadamente (pulo de duas) selecionadas até completar 36 domicílios em cada setor. Todas as mulheres de 20 a 69 anos residentes nesses domicílios foram convidadas a participar do estudo; no caso de recusa, foram realizadas mais duas tentativas em dias e horários diferentes. Foram excluídas gestantes e portadoras de déficit auditivo e/ou cognitivo. Especificamente para o presente artigo, foram incluídas apenas as mulheres na faixa etária de 20 a 60 anos idade, ou seja, foram excluídas mulheres acima de 60 anos por serem consideradas idosas e os critérios de definição de obesidade diferirem daqueles utilizados para adultos ${ }^{14}$.

Para investigar as características de interesse da amostra, foi aplicado um questionário padronizado, pré-codificado e pré-testado, por meio de entrevista domiciliar. Todos os entrevistadores foram submetidos a um programa de treinamento. Foi realizado estudo-piloto em outro setor censitário não incluído na pesquisa, cujo objetivo era testar os instrumentos e treinar os entrevistadores. A coleta de dados foi realizada no ano de 2015 , entre os meses de fevereiro e outubro.

Salienta-se que a maior parte das categorias das variáveis estudadas foi definida de forma a permitir comparabilidade com a literatura científica existente, em especial com estudo realizado com a mesma população-alvo no ano de $2003^{14}$. As variáveis demográficas investigadas foram: idade, referida 
no momento da entrevista e categorizada em faixas etárias de 10 anos; cor da pele, autorrelatada e categorizada em branca e não branca; situação conjugal, coletada e categorizada em solteira, casada/em união estável, divorciada/separada e viúva.

As variáveis socioeconômicas foram: escolaridade, referida em anos completos de estudo e categorizada em $\leq 4,5$ a 7,8 a 10 e $\geq 11$ anos; classe econômica, coletada segundo classificação da Associação Brasileira de Empresas de Pesquisa (ABEP) ${ }^{15}$ e categorizada em A, B, C e D-E; renda familiar per capita, criada pelo somatório da renda informada de cada componente da família no último mês, dividido pelo número de pessoas residentes no domicílio e pelo valor do salário mínimo da época $(\mathrm{R} \$ 788,00)$, categorizada em $\leq 1,1,01$ a 3, 3,01 a 6 e $\geq 6,01$ salários mínimos; estar trabalhando, informação referida sobre atividade remunerada no momento da entrevista, categorizado em não e sim.

As variáveis hereditária e reprodutiva foram: história familiar de obesidade, relatada e categorizada em não, mãe, pai e ambos; menarca, referida em anos completos, categorizada em 8 a 11, 12 a 13 e $\geq 14$ anos; número de gestações, referente ao número total de gestações durante sua vida reprodutiva (incluindo abortos, natimortos e filhos nascidos vivos), categorizado em nenhuma, 1 a 2,3 a 4 e $\geq 5$ gestações.

A variável comportamental prática de atividade física foi avaliada por meio do Questionário Internacional de Atividade Física (IPAQ), versão curta $^{16}$. Foram categorizadas como fisicamente inativas as mulheres que referiram praticar $<150$ minutos/semana de atividade física no lazer, de intensidade moderada ou vigorosa, e fisicamente ativas aquelas que referiram praticar $>150$ minutos/semana.

Os desfechos foram avaliados pelo cálculo do Índice de Massa Corporal (IMC), por ser um indicador amplamente utilizado em estudos clínicos e epidemiológicos para avaliação do estado nutricional ${ }^{3}$. O peso e a altura foram aferidos em duplicata, seguindo as recomendações do Ministério da Saúde (MS) ${ }^{17}$. O cálculo do IMC foi obtido pela divisão do peso (em quilogramas) pela altura (em metros quadrados). Foram classificadas com sobrepeso as mulheres com IMC entre 25,0 e $29,9 \mathrm{~kg} / \mathrm{m}^{2}$ e com obesidade aquelas com IMC $\geq 30,0 \mathrm{~kg} / \mathrm{m}^{2}$, segundo a $\mathrm{OMS}^{3}$.

A entrada dos dados foi realizada por meio do programa EpiData (versão 3.1), em dupla digitação e posterior comparação. As análises foram efetuadas no programa Stata (versão 13.0) e realizadas separadamente para cada um dos desfechos. As mulheres com obesidade foram excluídas da análise quando o desfecho considerado era sobrepeso. $\mathrm{Na}$ análise bivariada, foram realizados os testes de qui-quadrado de Pearson e tendência linear (variáveis ordinais).

Nas análises multivariadas, por se tratarem de desfechos frequentes na população, utilizou-se da regressão de Poisson com variância robusta. A análise ajustada seguiu um modelo conceitual definido a priori $i^{18}$. As variáveis que obtiveram associação com os desfechos a um nível de significância $\leq 20 \%$ na análise bruta foram incluídas no modelo multivariado como potenciais fatores de confusão. No bloco I, foram incluídas as demográficas e as socioeconômicas; no bloco II, as reprodutivas e a hereditária; e no bloco III, a comportamental. Mantiveram-se nos modelos ajustados apenas as variáveis com $p$ valor $\leq 0,20$. Escolaridade e classe econômica não foram ajustadas entre si nos modelos em razão da possibilidade de colinearidade entre essas variáveis. Nas análises bivariada e multivariada, as variáveis com $\mathrm{p}$ valor $<0,05$ foram consideradas associadas aos desfechos. O projeto foi submetido ao Comitê de Ética em Pesquisa da Universidade do Vale do Rio dos Sinos (Protocolo n 653.394-20/05/2014) e aprovado por ele.

\section{RESULTADOS}

Do total de 1.281 mulheres de 20 a 69 anos selecionadas para participar do estudo maior, houve 159 (11,9\%) perdas e recusas. Além disso, foram excluídas outras 141 (12,6\%), por possuírem mais de 60 anos. Assim, a amostra do presente estudo incluiu 981 mulheres entre 20 e 60 anos, com média de idade de 40,3 anos (DP $\pm 11,4)$.

A maioria das mulheres da amostra era branca $(74,1 \%)$ e casada ou vivendo em união estável $(64,6 \%)$. Cerca de metade da amostra tinha 11 anos ou mais de estudo, pertencia à classe econômica $\mathrm{C}$ e apresentava renda per capita de até um salário mínimo. Ademais, cerca de 2/3 delas estavam trabalhando no momento da entrevista. História familiar de obesidade foi presente em $44,2 \%$ das entrevistadas. Um elevado percentual de mulheres relatou ter tido uma ou duas gestações $(47,7 \%)$ e a grande maioria delas foi considerada fisicamente inativa $(85,8 \%)$ (Tabela 1$)$.

A prevalência de sobrepeso foi de 33\% (IC95\%: 30,1-36,0) e de obesidade foi de 31,2\% (IC95\%: 28,3-34,1). Maiores prevalências de sobrepeso foram verificadas nas mulheres de 40 a 49 anos, casadas/em união, com 5 a 7 anos de escolaridade, pertencentes à classe D-E, com renda de 1,01 a 3 salários mínimos, com mães obesas, com menarca acima dos 14 anos e com uma ou duas gestações. As maiores prevalências de obesidade foram encontradas nas mulheres com $\geq 50$ anos, viúvas, com baixa escolaridade, com pai e mãe obesos, com menarca precoce e que tiveram três ou mais gestações. As mulheres fisicamente ativas apresentaram a menor prevalência de obesidade $(19,4 \%)$, mas o sobrepeso foi elevado nesse grupo (Tabela 1).

$\mathrm{Na}$ análise ajustada, com o desfecho sobrepeso, verificou-se aumento da prevalência de sobrepeso conforme aumento da idade e menor classe econômica, sendo 1,82 vez maior (IC95\%: 1,39-2,39) para as mulheres de 50 a 60 anos e 2,09 vezes maior para as mulheres da classe D-E (IC95\%: 1,15-3,80). Além disso, a prevalência de sobrepeso também foi maior nas casadas ou vivendo em união estável (RP: 1,39; IC95\%: 1,10-1,76), naquelas com história familiar de mãe obesa (RP: 1,30; IC95\%: 1,09-1,54) e que tiveram uma ou mais gestações (RP: 1,77; IC95\%: 1,31-2,39) (Tabela 2).

Com o desfecho obesidade, verificou-se aumento da prevalência de obesidade conforme aumento da idade, sendo 1,83 vez maior (IC95\%: 1,31-2,54) para as mulheres de 
Tabela 1. Prevalências de sobrepeso e de obesidade segundo as características da amostra de mulheres residentes em São Leopoldo, Rio Grande do Sul, $2015(n=981)$

\begin{tabular}{|c|c|c|c|c|}
\hline Variáveis & n (\%) & $\begin{array}{c}\text { Prevalência de } \\
\text { sobrepeso }^{\text {a }} \\
\%\end{array}$ & $\begin{array}{c}\text { Prevalência de } \\
\text { obesidade }^{b} \\
\%\end{array}$ & $\mathbf{p}$ \\
\hline Faixa etária & & & & 0,001 \\
\hline 20-29 anos & $216(22,0)$ & 24,4 & 20,4 & \\
\hline 30-39 anos & $242(24,7)$ & 31,0 & 32,2 & \\
\hline $40-49$ anos & $274(27,9)$ & 39,1 & 32,5 & \\
\hline $50-60$ anos & $249(25,4)$ & 35,7 & 38,2 & \\
\hline Cor da pele & & & & 0,309 \\
\hline Branca & $727(74,1)$ & 34,4 & 30,5 & \\
\hline Não branca & $254(25,9)$ & 29,1 & 33,1 & \\
\hline Situação conjugal & & & & 0,001 \\
\hline Solteira & $214(21,8)$ & 25,7 & 25,2 & \\
\hline Casada/em união & $634(64,6)$ & 35,5 & 32,3 & \\
\hline Separada/divorciada & $94(9,6)$ & 34,0 & 34,0 & \\
\hline Viúva & $39(4,0)$ & 30,8 & 38,5 & \\
\hline Escolaridade (em anos) & & & & 0,001 \\
\hline$\geq 11$ & $437(44,5)$ & 32,5 & 24,9 & \\
\hline 8 a 10 & $181(18,5)$ & 31,5 & 33,7 & \\
\hline 5 a 7 & $215(21,9)$ & 36,7 & 37,2 & \\
\hline$\leq 4$ & $148(15,1)$ & 31,2 & 37,8 & \\
\hline Classe econômica $^{c}$ & & & & 0,015 \\
\hline A & $39(4,0)$ & 20,5 & 28,2 & \\
\hline B & $312(31,9)$ & 32,4 & 26,6 & \\
\hline $\mathrm{C}$ & $508(51,9)$ & 33,9 & 34,3 & \\
\hline $\mathrm{D}-\mathrm{E}$ & $119(12,2)$ & 35,3 & 30,3 & \\
\hline Renda per capita em SM ${ }^{d}$ & & & & 0,001 \\
\hline$\geq 6,01$ & $30(3,2)$ & 30,0 & 16,7 & \\
\hline 3,01 a 6 & $82(8,8)$ & 28,9 & 19,3 & \\
\hline 1,01 a 3 & $405(42,8)$ & 34,8 & 32,1 & \\
\hline$\leq 1$ & $429(45,3)$ & 32,2 & 33,8 & \\
\hline Trabalhando $^{\mathrm{e}}$ & & & & 0,219 \\
\hline Não & $377(38,5)$ & 31,6 & 34,5 & \\
\hline Sim & $603(61,5)$ & 33,8 & 29,2 & \\
\hline História familiar de obesidade & & & & 0,001 \\
\hline Não & $546(55,8)$ & 35,0 & 24,4 & \\
\hline Mãe & $238(24,3)$ & 35,3 & 38,7 & \\
\hline Pai & $81(8,3)$ & 28,4 & 29,6 & \\
\hline Ambos & $114(11,6)$ & 22,8 & 50,0 & \\
\hline Menarca & & & & $<\mathbf{0 , 0 0 1}$ \\
\hline 8 a 11 anos & $237(24,4)$ & 31,6 & 39,7 & \\
\hline 12 a 13 anos & $445(45,7)$ & 31,7 & 31,7 & \\
\hline$\geq 14$ anos & $291(29,7)$ & 35,7 & 23,7 & \\
\hline Número de gestações & & & & 0,001 \\
\hline Nenhuma & $162(16,5)$ & 22,8 & 14,2 & \\
\hline 1 a 2 & $468(47,7)$ & 38,5 & 29,1 & \\
\hline 3 a 4 & $258(26,3)$ & 28,7 & 43,4 & \\
\hline$\geq 5$ & $93(9,5)$ & 35,5 & 37,6 & \\
\hline Atividade física & & & & 0,003 \\
\hline Inativas $^{\mathrm{f}}$ & $842(85,8)$ & 32,7 & 33,1 & \\
\hline Ativas $\mathrm{g}$ & $139(14,2)$ & 35,3 & 19,4 & \\
\hline
\end{tabular}

SM: Salários mínimos; p: valor de p para o teste Qui-Quadrado; ${ }^{\mathrm{IMC}} 25-29,9 \mathrm{~kg} / \mathrm{m}^{2}$; ${ }^{\mathrm{b}} \mathrm{IMC} \geq 30,0 \mathrm{~kg} / \mathrm{m}^{2}$; ${ }^{\mathrm{C}}$ Critério de Classificação Econômica Brasil; ${ }^{\mathrm{a}}$ Salário mínimo ( $\mathrm{R} \$ 788,00)$ de janeiro de $2015 ;{ }^{\mathrm{e}}$ Trabalhando no momento da entrevista; ${ }^{\mathrm{f}}<150$ minutos/semana; ${ }^{\mathrm{g}}>150$ minutos/semana 
Tabela 2. Razões de prevalência (RP) brutas e ajustadas e IC95\% para sobrepeso segundo características demográficas, socioeconômicas, hereditária, reprodutivas e comportamentais de mulheres residentes de São Leopoldo, Rio Grande do Sul $(n=675)$

\begin{tabular}{|c|c|c|c|c|}
\hline Variáveis & RP Bruta (IC95\%) & p-valor & RP Ajustada (IC95\%) & p-valor \\
\hline Faixa etária & & $<0,001^{b}$ & & $<\mathbf{0 , 0 0 1} 1^{\mathrm{b}, \mathrm{c}}$ \\
\hline 20-29 anos & 1 & & 1 & \\
\hline 30-39 anos & $1,48(1,22-1,96)$ & & $1,43(1,08-1,91)$ & \\
\hline $40-49$ anos & $1,88(1,45-2,42)$ & & $1,83(1,41-2,28)$ & \\
\hline 50-60 anos & $1,88(1,44-2,44)$ & & $1,82(1,39-2,39)$ & \\
\hline Cor da pele & & $0,191^{\mathrm{a}}$ & & $0,156^{\mathrm{a}}$ \\
\hline Branca & 1 & & 1 & \\
\hline Não branca & $0,88(0,72-1,07)$ & & $0,87(0,72-1,05)$ & \\
\hline Situação conjugal & & $0,005^{a}$ & & $0,010^{\mathrm{a}, \mathrm{c}}$ \\
\hline Solteira & 1 & & 1 & \\
\hline Casada/em união & $1,52(1,21-1,92)$ & & $1,39(1,10-1,76)$ & \\
\hline Separada/divorciada & $1,50(1,09-2,07)$ & & $1,17(0,84-1,62)$ & \\
\hline Viúva & $1,45(0,92-2,29)$ & & $1,10(0,70-1,73)$ & \\
\hline Escolaridade (em anos) & & $0,018^{b}$ & & $0,975^{\mathrm{b}, \mathrm{e}}$ \\
\hline$\geq 11$ & 1 & & 1 & \\
\hline 8 a 10 & $1,10(0,87-1,37)$ & & $0,99(0,79-1,26)$ & \\
\hline 5 a 7 & $1,35(1,12-1,63)$ & & $1,09(0,88-1,34)$ & \\
\hline$\leq 4$ & $1,15(0,91-1,47)$ & & $0,94(0,72-1,23)$ & \\
\hline Classe econômica & & $0,021^{b}$ & & $\mathbf{0 , 0 0 1} 1^{\mathrm{b}, \mathrm{c}}$ \\
\hline A & 1 & & 1 & \\
\hline B & $1,54(0,84-2,82)$ & & $1,63(0,92-2,91)$ & \\
\hline $\mathrm{C}$ & $1,80(0,99-3,27)$ & & $2,00(1,13-3,54)$ & \\
\hline D-E & $1,77(0,95-3,30)$ & & $2,09(1,15-3,80)$ & \\
\hline Renda per capita em SM & & $0,120^{\mathrm{b}}$ & & $0,337^{\mathrm{b}}$ \\
\hline$\geq 6,01$ & 1 & & 1 & \\
\hline 3,01 a 6 & $0,99(0,54-1,84)$ & & $1,02(0,55-1,87)$ & \\
\hline 1,01 a 3 & $1,42(0,83-2,43)$ & & $1,35(0,79-2,33)$ & \\
\hline$\leq 1$ & $1,35(0,79-2,31)$ & & $1,27(0,72-2,26)$ & \\
\hline Trabalhando $^{f}$ & & $0,920^{\mathrm{a}}$ & - & \\
\hline Não & 1 & & & \\
\hline Sim & $0,99(0,84-1,17)$ & & & \\
\hline História familiar de obesidade & & $0,046^{a}$ & & $\mathbf{0 , 0 2 2 ^ { \mathrm { a } , \mathrm { d } }}$ \\
\hline Não & 1 & & 1 & \\
\hline Mãe & $1,24(1,04-1,48)$ & & $1,30(1,09-1,54)$ & \\
\hline Pai & $0,87(0,62-1,22)$ & & $0,96(0,69-1,32)$ & \\
\hline Ambos & $0,99(0,73-1,33)$ & & $1,03(0,78-1,38)$ & \\
\hline Menarca & & $0,350^{\mathrm{b}}$ & - & \\
\hline 8 a 11 & 1 & & & \\
\hline 12 a 13 anos & $0,88(0,72-1,08)$ & & & \\
\hline$\geq 14$ ou mais & $0,89(0,72-1,10)$ & & & \\
\hline Número de gestações & & $<0,001^{\mathrm{a}}$ & & $\mathbf{0 , 0 0 2 ^ { \mathrm { a } , \mathrm { d } }}$ \\
\hline Nenhuma & 1 & & 1 & \\
\hline 1 a 2 & $2,04(1,52-2,73)$ & & $1,77(1,31-2,39)$ & \\
\hline 3 a 4 & $1,90(1,38-2,62)$ & & $1,50(1,07-2,10)$ & \\
\hline$\geq 5$ & $2,14(1,50-3,05)$ & & $1,61(1,11-2,33)$ & \\
\hline Atividade física & & $0,341^{\mathrm{a}}$ & - & \\
\hline Inativas & 1 & & & \\
\hline Ativas & $0,89(0,71-1,12)$ & & & \\
\hline
\end{tabular}

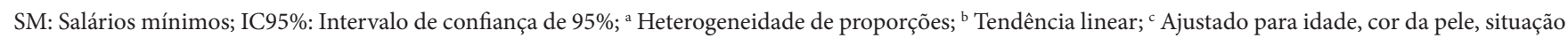
conjugal e classe econômica; ${ }^{\mathrm{d}}$ Ajustado para as variáveis anteriores mais história familiar de obesidade e número de gestações; ${ }^{\mathrm{e}}$ Não ajustado para variável classe econômica; ${ }^{\mathrm{f}}$ Trabalhando no momento da entrevista 
50 a 60 anos de idade. Constatou-se diminuição da prevalência de obesidade quanto maior a idade de menarca, sendo 0,62 vez menor para aquelas com menarca acima de 14 anos. Além disso, a prevalência de obesidade foi maior nas mulheres com história familiar de mãe obesa e de ambos (pai e mãe) obesos
(RP: 1,95; IC95\%: 1,52-2,50) e que tiveram três ou quatro gestações (RP: 2,28; IC95\%: 1,48-3,50). Mulheres consideradas fisicamente ativas apresentaram menor prevalência (RP: 0,68; IC95\%: 0,48-0,96) de obesidade se comparadas aquelas que eram fisicamente inativas (Tabela 3 ).

Tabela 3. Razões de prevalência (RP) brutas e ajustadas e IC95\% para obesidade segundo características demográficas, socioeconômicas, hereditária, reprodutivas e comportamentais de mulheres residentes de São Leopoldo, Rio Grande do Sul $(n=981)$

\begin{tabular}{|c|c|c|c|c|}
\hline Variáveis & RP Bruta (IC95\%) & p-valor & RP Ajustada (IC95\%) & p-valor \\
\hline Faixa etária & & $<0,001^{b}$ & & $0,001^{b, c}$ \\
\hline 20-29 anos & 1 & & 1 & \\
\hline 30-39 anos & $1,58(1,15-2,18)$ & & $1,60(1,16-2,22)$ & \\
\hline 40-49 anos & $1,59(1,16-2,18)$ & & $1,52(1,10-2,13)$ & \\
\hline $50-60$ anos & $1,87(1,38-2,55)$ & & $1,83(1,31-2,54)$ & \\
\hline Cor da pele & & $0,449^{a}$ & - & \\
\hline Branca & 1 & & & \\
\hline Não branca & $1,08(0,88-1,33)$ & & & \\
\hline Situação conjugal & & $0,170^{\mathrm{a}}$ & & $0,684^{\mathrm{a}}$ \\
\hline Solteira & 1 & & 1 & \\
\hline Casada/em união & $1,28(0,99-1,66)$ & & $1,18(0,90-1,54)$ & \\
\hline Separada/divorciada & $1,35(0,94-1,94)$ & & $1,14(0,78-1,67)$ & \\
\hline Viúva & $1,52(0,96-2,41)$ & & $1,18(0,73-1,90)$ & \\
\hline Escolaridade (em anos) & & $<0,001^{b}$ & & $0,152^{\mathrm{b}, \mathrm{e}}$ \\
\hline$\geq 11$ & 1 & & 1 & \\
\hline 8 a 10 & $1,35(1,04-1,75)$ & & $1,28(0,98-1,68)$ & \\
\hline 5 a 7 & $1,49(1,17-1,89)$ & & $1,30(0,99-1,70)$ & \\
\hline$\leq 4$ & $1,52(1,16-1,97)$ & & $1,26(0,94-1,70)$ & \\
\hline Classe econômica & & $0,140^{\mathrm{b}}$ & & $0,866^{\mathrm{b}, \mathrm{f}}$ \\
\hline A & 1 & & 1 & \\
\hline B & $0,94(0,55-1,61)$ & & $0,89(0,50-1,58)$ & \\
\hline $\mathrm{C}$ & $1,21(0,72-2,03)$ & & $1,02(0,57-1,83)$ & \\
\hline $\mathrm{D}-\mathrm{E}$ & $1,07(0,61-1,90)$ & & $0,88(0,46-1,71)$ & \\
\hline Renda per capita em SM & & $0,006^{\mathrm{b}}$ & & $0,023^{b, c}$ \\
\hline$\geq 6,01$ & 1 & & 1 & \\
\hline 3,01 a 6 & $1,16(0,46-2,89)$ & & $1,16(0,46-2,88)$ & \\
\hline 1,01 a 3 & $1,93(0,85-4,34)$ & & $1,86(0,81-4,24)$ & \\
\hline$\leq 1$ & $2,03(0,90-4,57)$ & & $1,86(0,81-4,28)$ & \\
\hline Trabalhando ${ }^{g}$ & & $0,080^{\mathrm{a}}$ & & $0,772^{\mathrm{a}}$ \\
\hline Não & 1 & & 1 & \\
\hline Sim & $0,85(0,70-1,02)$ & & $0,97(0,80-1,18)$ & \\
\hline História familiar de obesidade & & $<0,001^{\text {a }}$ & & $<0,001^{\text {a,d }}$ \\
\hline Não & 1 & & 1 & \\
\hline Mãe & $1,59(1,28-1,97)$ & & $1,47(1,18-1,82)$ & \\
\hline Pai & $1,22(0,84-1,75)$ & & $1,21(0,83-1,77)$ & \\
\hline Ambos & $2,05(1,62-2,60)$ & & $1,95(1,52-2,50)$ & \\
\hline Menarca & & $<0,001^{b}$ & & $<\mathbf{0 , 0 0 1} 1^{\mathrm{b}, \mathrm{d}}$ \\
\hline 8 a 11 anos & 1 & & 1 & \\
\hline 12 a 13 anos & $0,80(0,65-0,98)$ & & $0,83(0,68-1,02)$ & \\
\hline$\geq 14$ & $0,60(0,46-0,77)$ & & $0,62(0,48-0,81)$ & \\
\hline Número de gestações & & $<0,001^{b}$ & & $0,001^{b, d}$ \\
\hline Nenhuma & 1 & & 1 & \\
\hline 1 a 2 & $2,05(1,37-3,07)$ & & $1,72(1,14-2,58)$ & \\
\hline 3 a 4 & $3,06(2,04-4,58)$ & & $2,28(1,48-3,50)$ & \\
\hline$\geq 5$ & $2,65(1,67-4,20)$ & & $1,92(1,16-3,18)$ & \\
\hline Atividade física & & $0,003^{a}$ & & $0,028^{a, d}$ \\
\hline Inativas & 1 & & 1 & \\
\hline Ativas & $0,59(0,41-0,83)$ & & $0,68(0,48-0,96)$ & \\
\hline
\end{tabular}

SM: Salários mínimos; IC95\%: Intervalo de confiança de $95 \%$; ${ }^{a}$ Heterogeneidade de proporções; ${ }^{\mathrm{b}}$ Tendência linear; ${ }^{\mathrm{c}}$ Ajustado para idade, escolaridade e renda;

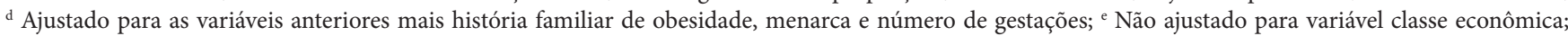

${ }^{\mathrm{f}}$ Não ajustado para variável escolaridade; ${ }^{\mathrm{g}}$ Trabalhando no momento da entrevista 


\section{DISCUSSÃO}

Verificou-se elevada prevalência de sobrepeso e de obesidade em uma amostra representativa de mulheres de 20 a 60 anos de idade residentes na região urbana de São Leopoldo/RS. Características como idade, história familiar de obesidade e número de gestações estiveram associadas a ambos os eventos. Entretanto, ser casada ou viver em união estável e pertencer à classe $\mathrm{C}$ ou inferior (D-E) foram características relacionadas apenas com sobrepeso; por sua vez, ter baixa renda, menarca precoce e inatividade física estiveram associadas apenas com obesidade.

Verificou-se que $2 / 3$ da amostra estava acima do peso recomendado. Esse é um dado extremamente preocupante, uma vez que o sobrepeso e a obesidade são reconhecidos fatores de risco para o aumento de morbidades e mortalidade ${ }^{6}$, além do impacto financeiro sobre o sistema de saúde ${ }^{19}$.

A prevalência de obesidade na população feminina está aumentando progressivamente nas últimas décadas. Segundo dados de um estudo realizado em 200 países, a prevalência passou de 6,4\% (IC95\%: 5,1-7,8) em 1975 para 14,9\% (IC95\%: 13,6-16,1) em $2014^{4}$. No Brasil, a prevalência aumentou de 13,1\% em 2002-2003 para 16,9\% em 2008-2009 ${ }^{12}$. Em 2016, 20,3\% (IC95\%: 17,3-23,2) da população feminina de Porto Alegre apresentava obesidade, ocupando o $19^{\circ}$ lugar entre as capitais do Brasil e o Distrito Federal com maior prevalência de obesidade ${ }^{20}$.

Fazendo uma análise comparativa entre o presente estudo e outro de base populacional realizado em 2003, também com amostra representativa de mulheres de 20 a 60 anos de idade de São Leopoldo ${ }^{14}$, evidenciou-se o rápido aumento da obesidade, de $18 \%$ no ano de 2003 para 31,2\% em 2015. Por outro lado, a prevalência de sobrepeso manteve-se estável, de 31,7\% em 2003 para 33\% em 2015.

Essas situações podem ser mais bem avaliadas quando se observam as tendências por faixas etárias. Comparando os dois estudos de 2003 e 2015, nota-se que os maiores incrementos de obesidade ocorreram nas mulheres mais jovens, das faixas etárias de 20 a 29 anos (de 9,6\% para 20,4\%) e de 30 a 39 anos (de $12,8 \%$ para $32,2 \%$ ), ou seja, acréscimos de $114 \%$ e $151 \%$, respectivamente. Nas mulheres mais velhas, o aumento ocorreu de forma mais branda (16,5\%), especialmente na faixa etária de 50 a 60 anos. Por outro lado, um dado novo refere-se à redução nas prevalências de sobrepeso em todas as faixas etárias (reduções variando de $8 \%$ até $28 \%$ ). A prevalência de obesidade desta pesquisa também foi superior do que a encontrada (29,2\%) no estudo de base populacional realizado em Pelotas/RS, no ano de $2010^{21}$.

Salienta-se que, neste estudo, assim como em outros com similar delineamento, a idade manteve-se associada aos desfechos, mesmo ajustada para diversos fatores de confusão $0^{14,22-24}$. A associação entre idade e sobrepeso ou obesidade pode ser parcialmente explicada pelo declínio da taxa metabólica basal e diminuição da massa muscular, que fisiologicamente acompanha o processo de envelhecimento, bem como pela redução natural da prática atividade física ${ }^{3,25-27}$

As mulheres casadas tiveram maior prevalência de sobrepeso. Uma possível explicação é o fato de que o matrimônio ocasiona mudanças tanto no estilo de vida quanto nos hábitos alimentares, o que pode contribuir para o ganho de peso corporal ${ }^{28}$. Um estudo constatou que a prevalência de sobrepeso foi $19 \%$ maior nas mulheres casadas em comparação com as solteiras ${ }^{29}$. Com Teichmann et al. ${ }^{14}$, esse índice subiu para 42\% (RP: 1,42; IC95\%: 1,05-1,57).

A classe econômica apresentou associação inversa com o sobrepeso. Não foram encontrados estudos com resultados semelhantes em relação ao desfecho sobrepeso, apenas para obesidade. Na pesquisa conduzida em São Leopoldo/RS no ano de 2003, a prevalência de obesidade foi 3,36 vezes maior nas mulheres pertencentes à classe econômica $\mathrm{D}$-E, em comparação com aquelas pertencentes à classe econômica $\mathrm{A}^{14}$.

A renda familiar, por sua vez, não apresentou associação com sobrepeso, mas houve aumento na prevalência de obesidade para as mulheres com menor renda. A associação de renda familiar com sobrepeso e obesidade não tem resultados consistentes entre os diversos estudos com amostras de base populacional realizados no Sul do Brasil nos últimos 20 anos, pois alguns verificaram associações significativas ${ }^{14}$, e outros, não ${ }^{21,30,31}$.

A ausência de associação entre escolaridade com os desfechos foi um resultado inesperado, pois a baixa escolaridade tem sido amplamente descrita na literatura como fator associado ao sobrepeso e à obesidade em mulheres ${ }^{14,22,29,32}$. Isso poderia ser parcialmente esclarecido pelo efeito positivo da escolaridade, que repercute no acesso à informação de qualidade relacionada à saúde e na maior busca de serviços e de profissionais de saúde, o que auxilia na adesão a hábitos alimentares mais saudáveis. Além disso, a escolaridade está relacionada aos determinantes mais precoces do ciclo de vida e tende a influenciar na ocupação, renda e classe econômica ${ }^{21,26,33,34}$. Contudo, não foi o resultado apresentado nesta pesquisa.

Os resultados encontrados da relação de renda familiar per capita e da escolaridade com os desfechos deste estudo conduzem a pensar que, provavelmente, novas abordagens, que incluam a investigação do ambiente alimentar e o acesso a alimentos, sejam necessárias para complementar a compreensão sobre a ocorrência do sobrepeso e da obesidade na população ${ }^{35,36}$.

Observou-se aumento na prevalência de sobrepeso e obesidade nas mulheres que tinham mãe obesa. Adicionalmente, o aumento da prevalência de obesidade foi maior quando ambos os pais eram obesos. Esse efeito também foi verificado em outros estudos ${ }^{14,24,30,37}$, possivelmente indicando a ação de fatores genéticos, além de exposição compartilhada aos fatores comportamentais, ambientais e culturais ${ }^{37,38}$. 
A menarca mostrou associação linear inversa com a obesidade. A mesma tendência foi verificada em outras pesquisas ${ }^{14,29,39,40}$. Vários fatores têm sido identificados como mecanismo de disparo do início da puberdade, por exemplo, a síntese de leptina pelos adipócitos, acelerando a maturação do tecido e a função reprodutiva ${ }^{41-43}$. Nessa perspectiva, a menarca precoce (inferior a 12 anos de idade) pode ser apontada como um marcador de risco, sendo o IMC acima do recomendado na infância um preditor da menarca precoce ${ }^{44-49}$.

A prevalência de obesidade foi maior nas mulheres que já passaram por uma ou duas gestações, ampliando para aquelas com três ou quatro. Resultados similares foram apontados em outros estudos ${ }^{14,29}$. Essa associação pode ser compreendida pelo ganho de peso gestacional superior ao preconizado pelo Institute of Medicine (IOM), bem como pela não adesão ao aleitamento materno segundo as recomendações da OMS, favorecendo a retenção de peso pós-parto ${ }^{50-54}$. Segundo metanálise, cada mês de aleitamento materno reduz em $4 \%$ as chances de excesso de peso, demonstrando associação dose-resposta entre maior duração da amamentação e diminuição da retenção de peso ${ }^{55}$. Relacionam-se também com retenção de peso pós-parto o excesso de peso pré-gestacional, o consumo de calorias em excesso e o baixo nível de atividade física no período gestacional e pós-parto, assim como o tempo pequeno entre as gestações ${ }^{53}$.

A associação entre atividade física e obesidade também foi verificada em um estudo conduzido na Espanha ${ }^{56}$. Mulheres que praticavam $\geq 150$ minutos/semana de atividade física (fisicamente ativas) tiveram redução de $28 \%$ nas chances de obesidade em comparação às fisicamente inativas. Essa associação pode ser compreendida pelo efeito positivo da atividade física, sendo o principal determinante de gasto calórico. A atividade física estimula a resposta termogênica via aumento da taxa metabólica em repouso, com consequente elevação do gasto energético, aumentando a capacidade de mobilização e oxidação de tecido adiposo e favorecendo a perda de peso corporal ${ }^{57}$.

Os achados devem ser interpretados considerando a limitação inerente aos estudos transversais, ou seja, a possibilidade de viés de causalidade reversa. Por outro lado, destaca-se o rigor metodológico com que foram conduzidas todas as etapas do estudo, bem como a taxa de resposta (cerca de 90\%). Salienta-se que o percentual de mulheres nas faixas etárias deste estudo é semelhante ao encontrado no último censo demográfico na cidade de São Leopoldo ${ }^{58}$, indicando que o processo de amostragem foi conduzido de modo a conferir representatividade. Além disso, a análise multivariada foi realizada com o intuito de controlar os fatores de confusão, e toda a modelagem foi realizada com base no modelo conceitual definido a priori.

Verificaram-se diferenciais entre as características da amostra e a ocorrência de sobrepeso e de obesidade. Se, por um lado, isso reforçou a multicausalidade envolvida nesses eventos de saúde, por outro possibilitou a visualização do agravamento do problema da obesidade, principalmente pelo abrupto aumento em "mulheres adultas jovens". Ademais, os achados desta pesquisa evidenciaram a forte relação dos desfechos com idade, características da vida reprodutiva e história familiar de obesidade, ou seja, podem ser norteadores para subsidiar o planejamento e a implementação de políticas e programas direcionados para ações de prevenção de longo prazo e voltadas concomitantemente para mulheres em diferentes etapas do ciclo da vida.

\section{REFERÊNCIAS}

1. World Health Organization. Diet, nutrition and the prevention of chronic diseases: Report FAO/WHO Expert Consulation. Geneva: WHO; 2003.

2. World Health Organization. Preventing chronic diseases: a vital investment. Geneva: WHO; 2005.

3. World Health Organization. Obesity: preventing and managing the global epidemic. Geneva: WHO; 2000.

4. NCD-RisC. Trends in adult body-mass index in 200 countries from 1975 to 2014: a pooled analysis of 1698 population-based measurement studies with 19.2 million participants. Lancet. 2016;387(10026):1377-96. http:// dx.doi.org/10.1016/S0140-6736(16)30054-X. PMid:27115820.

5. World Health Organization. Global status report on noncommunicable diseases 2014. Geneva: WHO; 2014.

6. World Health Organization. Global health risks: mortality and burden of disease attributable to selected major risks. Geneva: WHO; 2009.

7. Callaway LK, Chang AM, McIntyre HD, Prins JB. The prevalence and impact of overweight and obesity in an Australian obstetric population. Med J Aust. 2006;184(2):56-9. http://dx.doi.org/10.5694/j.1326-5377.2006. tb00115.x. PMid:16411868.
8. Masturzo B, Franze V, Germano C, Attini R, Gennarelli G, Lezo A, et al. Risk of adverse pregnancy outcomes by pre-pregnancy Body Mass Index among Italian population: a retrospective population-based cohort study on 27,807 deliveries. Arch Gynecol Obstet. 2019;299(4):983-91. http:// dx.doi.org/10.1007/s00404-019-05093-0. PMid:30770990.

9. Kuba VM, Cavalieri PM, Christóforo ÂC, Faria R Jr, Caetano R, Coeli $\mathrm{CM}$, et al. Resistência insulínica e perfil metabólico em pacientes com síndrome dos ovários policísticos de peso normal e sobrepeso/obesidade. Arq Bras Endocrinol Metabol. 2006;50(6):1026-33. http://dx.doi.org/10.1590/ S0004-27302006000600008. PMid:17221108.

10. Wang JX, Davies MJ, Norman RJ. Obesity increases the risk of spontaneous abortion during infertility treatment. Obes Res. 2002;10(6):551-4. http:// dx.doi.org/10.1038/oby.2002.74. PMid:12055331.

11. Ramoniene G, Maleckiene L, Nadisauskiene RJ, Bartuseviciene E, Railaite DR, Maciuleviciene R, et al. Maternal obesity and obstetric outcomes in a tertiary referral center. Medicina (B Aires). 2017;53(2):109-13. http:// dx.doi.org/10.1016/j.medici.2017.03.003. PMid:28462873.

12. Instituto Brasileiro de Geografia e Estatística. Pesquisa de Orçamentos Familiares 2002-2003: análise da disponibilidade domiciliar de alimentos e do estado nutricional do Brasil. Rio de Janeiro: IBGE; 2004. 
13. Instituto Brasileiro de Geografia e Estatística. Pesquisa de Orçamentos Familiares 2008-2009: antropometria e estado nutricional de crianças, adolescentes e adultos no Brasil. Rio de Janeiro: IBGE; 2010.

14. Teichmann L, Olinto MTA, Costa JSD, Ziegler D. Fatores de risco associados ao sobrepeso e a obesidade em mulheres de São Leopoldo, RS. Rev Bras Epidemiol. 2006;9(3):360-73. http://dx.doi.org/10.1590/S1415$790 X 2006000300010$

15. Associação Brasileira de Empresas de Pesquisa. Critério de Classificação Econômica Brasil [Internet]. São Paulo: ABEP; 2018 [citado em 2018 mar 14]. Disponível em: http://www.abep.org/criterio-brasil

16. Matsudo S, Araújo T, Matsudo V, Andrade D, Andrade E, Oliveira LC, et al. Questionário Internacional de Atividade Física (IPAQ): Estudo de Validade e Reprodutibilidade no Brasil. Rev Bras Ativ Fis Saude. 2001;6(2):5-18.

17. Brasil. Ministério da Saúde. Secretaria de Atenção à Saúde. Departamento de Atenção Básica. Orientações para a coleta e análise de dados antropométricos em serviços de saúde: Norma Técnica do Sistema de Vigilância Alimentar e Nutricional - SISVAN. Brasília: Ministério da Saúde; 2011.

18. Victora CG, Huttly SR, Fuchs SC, Olinto MT. The role of conceptual frameworks in epidemiological analysis: a hierarchical approach. Int J Epidemiol. 1997;26(1):224-7. http://dx.doi.org/10.1093/ije/26.1.224. PMid:9126524.

19. Bahia L, Coutinho ES, Barufaldi LA, Abreu GA, Malhão TA, de Souza CP, et al. The costs of overweight and obesity-related diseases in the Brazilian public health system: cross-sectional study. BMC Public Health. 2012;12(440):440. http://dx.doi.org/10.1186/1471-2458-12-440. PMid:22713624.

20. Brasil. Ministério da Saúde. Secretaria de Vigilância em Saúde. Departamento de Vigilância de Doenças e Agravos não Transmissíveis e Promoção da Saúde. Vigitel Brasil 2016: vigilância de fatores de risco e proteção para doenças crônicas por inquérito telefônico. Brasília: Ministério da Saúde; 2017.

21. Linhares RS, Horta BL, Gigante DP, Dias-da-Costa JS, Olinto MT. Distribuição de obesidade geral e abdominal em adultos de uma cidade no Sul do Brasil. Cad Saude Publica. 2012;28(3):438-47. http://dx.doi.org/10.1590/S0102311X2012000300004. PMid:22415176.

22. Ferreira RAB, Benicio MHDA. Obesidade em mulheres brasileiras: associação com paridade e nível socioeconômico. Rev Panam Salud Publica. 2015;37(4/5):337-42. PMid:26208205.

23. Paul E, Mtumwa AH, Ntwenya JE, Vuai SA. Disparities in risk factors associated with obesity between Zanzibar and Tanzania Mainland among women of reproductive age based on the 2010 TDHS. J Obes. 2016:1420673. http://dx.doi.org/10.1155/2016/1420673. PMID: 27721990.

24. Wang H, Wang J, Liu MM, Wang D, Liu YQ, Zhao Y, et al. Epidemiology of general obesity, abdominal obesity and related risk factors in urban adults from 33 communities of Northeast China: the CHPSNE study. BMC Public Health. 2012;12(1):967. http://dx.doi.org/10.1186/1471-2458-12-967. PMid:23146089.

25. Zeinali F, Habibi N, Samadi M, Azam K, Djafarian K. Relation between lifestyle and socio-demographic factors and body composition among the elderly. Glob J Health Sci. 2016;8(8):53715. PMid:27045408.

26. Pinho CPS, Diniz AS, Arruda IK, Lira PI, Sequeira LA, Gonçalves FC, et al. Excesso de peso em adultos do Estado de Pernambuco, Brasil: magnitude e fatores associados. Cad Saude Publica. 2011;27(12):2340-50. http://dx.doi. org/10.1590/S0102-311X2011001200006. PMid:22218577.

27. Ronsoni RM, Pereira MR, Silva RH, Becker IC, Sehnen L Jr. Prevalência de obesidade e seus fatores associados na população de Tubarão-SC. ACM Arq Catarin Med. 2005;34(3):51-7.
28. Moraes SA, Humberto JSM, Freitas ICM. Estado nutricional e fatores sociodemográficos em adultos residentes em Ribeirão Preto, SP, 2006: projeto OBEDIARP. Rev Bras Epidemiol. 2011;14(4):662-76. http://dx.doi. org/10.1590/S1415-790X2011000400013. PMid:22218665.

29. Correia LL, Silveira DMI, Silva AC, Campos JS, Machado MMT, Rocha HAL, et al. Prevalência e determinantes de obesidade e sobrepeso em mulheres em idade reprodutiva residentes na região semiárida do Brasil. Cien Saude Colet. 2011;16(1):133-45. http://dx.doi.org/10.1590/S141381232011000100017. PMid:21180822.

30. Gigante DP, Barros FC, Post CLA, Olinto MTA. Prevalência de obesidade em adultos e seus fatores de risco. Rev Saude Publica. 1997;31(3):236-46. http://dx.doi.org/10.1590/S0034-89101997000300004. PMid:9515260.

31. Gigante DP, Dias-da-Costa JS, Olinto MTA, Menezes AMB, Silvia M. Obesidade da população adulta de Pelotas, Rio Grande do Sul, Brasil e associação com nível sócio-econômico. Cad Saude Publica. 2006;22(9):18739. http://dx.doi.org/10.1590/S0102-311X2006000900018. PMid:16917584.

32. Cheng H, Montgomery S, Green A, Furnham A. Biomedical, psychological, environmental and behavioural factors associated with adult obesity in a nationally representative sample. J Public Health (Oxf). In press 2019. http://dx.doi.org/10.1093/pubmed/fdz009. PMid:30799484.

33. Devaux M, Sassi F, Cecchini M, Borgonovi F, Church J. Exploring the relationship between education and obesity. OECD Journal. 2011;2011(1):12159. http://dx.doi.org/10.1787/eco_studies-2011-5kg5825v1k23.

34. Velásquez-Meléndez G, Pimenta AM, Kac G. Epidemiologia do sobrepeso e da obesidade e seus fatores determinantes em Belo Horizonte (MG), Brasil: estudo transversal de base populacional. Rev Panam Salud Publica. 2004;16(5):308-14. http://dx.doi.org/10.1590/S1020-49892004001100003. PMid:15729979.

35. Feng J, Glass TA, Curriero FC, Stewart WF, Schwartz BS. The built environment and obesity: a systematic review of the epidemiologic evidence. Health Place. 2010;16(2):175-90. http://dx.doi.org/10.1016/j. healthplace.2009.09.008. PMid:19880341.

36. Fuller D, Cummins S, Matthews SA. Does transportation mode modify associations between distance to food store, fruit and vegetable consumption, and BMI in low-income neighborhoods? Am J Clin Nutr. 2013;97(1):16772. http://dx.doi.org/10.3945/ajen.112.036392. PMid:23193006.

37. Vedana EHB, Peres MA, Neves J, Rocha GC, Longo GZ. Prevalência de obesidade e fatores potencialmente causais em adultos em região do sul do Brasil. Arq Bras Endocrinol Metabol. 2008;52(7):1156-62. http://dx.doi. org/10.1590/S0004-27302008000700012. PMid:19082304.

38. Doerr M, Teng K. Family history: still relevant in the genomics era. Cleve Clin J Med. 2012;79(5):331-6. http://dx.doi.org/10.3949/ccjm.79a.11065. PMid:22550075.

39. Sinha I, Mondal N, Sen J. Effects of socio-economic, demographic and lifestyle variables on overweight and obesity among rural Rajbanshi postmenopausal women of India. Anthropol Anz. 2018;75(3):251-62. http:// dx.doi.org/10.1127/anthranz/2018/0863. PMid:29892775.

40. Theodoro H, Rodrigues AD, Mendes KG, Liane RH, Paniz VM, Olinto MT. Reproductive characteristics and obesity in middle-aged women seen at an outpatient clinic in southern Brazil. Menopause. 2012;19(9):1022-8. http://dx.doi.org/10.1097/gme.0b013e3182503834. PMid:22549168.

41. Ahima RS, Dushay J, Flier SN, Prabakaran D, Flier JS. Leptin accelerates the onset of puberty in normal female mice. J Clin Invest. 1997;99(3):391-5. http://dx.doi.org/10.1172/JCI119172. PMid:9022071. 
42. Gavela-Perez T, Navarro P, Soriano-Guillen L, Garces C. High prepubertal leptin levels are associated with earlier menarcheal age. J Adolesc Health. 2016;59(2):177-81. http://dx.doi.org/10.1016/j.jadohealth.2016.03.042. PMid:27297138.

43. DiVall SA, Radovick S. Pubertal development and menarche. Ann N Y Acad Sci. 2008;1135(1):19-28. http://dx.doi.org/10.1196/annals.1429.026. PMid:18574204.

44. Davison KK, Susman EJ, Birch LL. Percent body fat at age 5 predicts earlier pubertal development among girls at age 9. Pediatrics. 2003;111(4 Pt 1):815-21. http://dx.doi.org/10.1542/peds.111.4.815. PMid:12671118.

45. Euling SY, Herman-Giddens ME, Lee PA, Selevan SG, Juul A, Sorensen TI, et al. Examination of US puberty-timing data from 1940 to 1994 for secular trends: panel findings. Pediatrics. 2008;121(Suppl 3):S172-91. http:// dx.doi.org/10.1542/peds.2007-1813D. PMid:18245511.

46. Frontini MG, Srinivasan SR, Berenson GS. Longitudinal changes in risk variables underlying metabolic Syndrome X from childhood to young adulthood in female subjects with a history of early menarche: the Bogalusa Heart Study. Int J Obes Relat Metab Disord. 2003;27(11):1398-404. http:// dx.doi.org/10.1038/sj.ijo.0802422. PMid:14574352.

47. Barcellos Gemelli IF, Farias EDS, Souza OF. Age at Menarche and Its Association with Excess Weight and Body Fat Percentage in Girls in the Southwestern Region of the Brazilian Amazon. J Pediatr Adolesc Gynecol. 2016;29(5):482-8. http://dx.doi.org/10.1016/j.jpag.2016.02.011. PMid:26964519.

48. Lian Q, Mao Y, Luo S, Zhang S, Tu X, Zuo X, et al. Puberty timing associated with obesity and central obesity in Chinese Han girls. BMC Pediatr. 2019;19(1):1. http://dx.doi.org/10.1186/s12887-018-1376-4. PMid:30606158.

49. Kivimèki M, Lawlor DA, Smith GD, Elovainio M, Jokela M, KeltikangasJèrvinen L, et al. Association of age at menarche with cardiovascular risk factors, vascular structure, and function in adulthood: the Cardiovascular Risk in Young Finns study. Am J Clin Nutr. 2008;87(6):1876-82. http:// dx.doi.org/10.1093/ajcn/87.6.1876. PMid:18541580.

50. Forte CC, Bernardi JR, Goldani MZ, Bosa VL. Relação entre a retenção de peso nos primeiros três meses pós-parto com ganho de peso e ingestão alimentar durante a gestação. Rev Bras Saúde Mater Infant. 2015;15(3):27987. http://dx.doi.org/10.1590/S1519-38292015000300003.

51. Santos S, Voerman E, Amiano P, Barros H, Beilin LJ, Bergstrom A, et al. Impact of maternal body mass index and gestational weight gain on pregnancy complications: An individual participant data meta-analysis of European, North American and Australian cohorts. BJOG. 2019;126(8):984-95. http:// dx.doi.org/10.1111/1471-0528.15661. PMid:30786138.

52. Institute of Medicine and National Research Council. Weight gain during pregnancy: reexamining the guidelines. Washington, DC: The National Academies Press; 2009.

53. Zanotti J, Capp E, Wender MCO. Factors associated with postpartum weight retention in a Brazilian cohort. Rev Bras Ginecol Obstet. 2015;37(4):164-71. http://dx.doi.org/10.1590/SO100-720320150005186. PMid:25992499.

54. Brasil. Ministério da Saúde. Secretaria de Atenção à Saúde. Departamento de Atenção Básica. Saúde da criança: aleitamento materno e alimentação complementar. Brasília: Ministério da Saúde; 2015.

55. Harder T, Bergmann R, Kallischnigg G, Plagemann A. Duration of breastfeeding and risk of overweight: a meta-analysis. Am J Epidemiol. 2005;162(5):397-403. http://dx.doi.org/10.1093/aje/kwi222. PMid:16076830.

56. Romo-Perez V, Souto D, Mota J. Walking, body mass index, and self-rated health in a representative sample of Spanish adults. Cad Saude Publica. 2016;32(1). http://dx.doi.org/10.1590/0102-311X00166414. PMid:26886367.

57. McMurray RG, Hackney AC. Interactions of metabolic hormones, adipose tissue and exercise. Sports Med. 2005;35(5):393-412. http://dx.doi. org/10.2165/00007256-200535050-00003. PMid:15896089.

58. Instituto Brasileiro de Geografia e Estatística. Censo Demográfico 2010 Cidades@: São Leopoldo/RS [Internet]. 2010 [citado em 2018 Mar 14]. Disponível em: https://cidades.ibge.gov.br/brasil/rs/sao-leopoldo/panorama

Recebido em: Jun. 04, 2018 Aprovado em: Mar. 29, 2019 